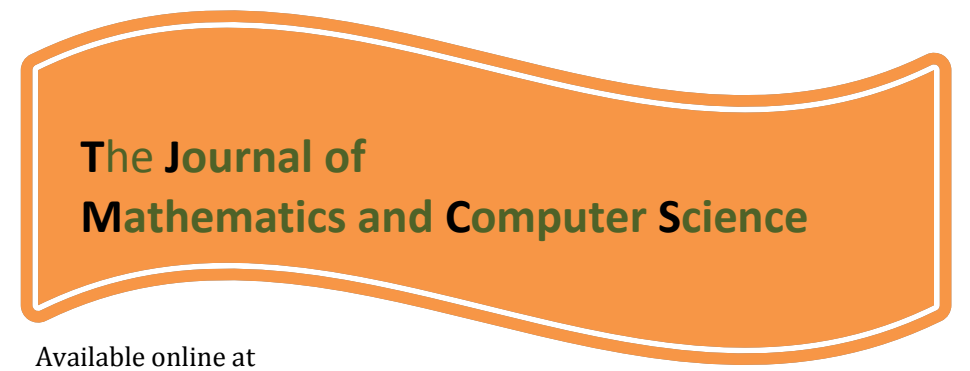

http://www.TJMCS.com

The Journal of Mathematics and Computer Science Vol. 4 No.3 (2012) 448 - 455

\title{
Solving an Elliptic Optimal Control Problem with BEM and FEM
}

\author{
Ali Zakeri, ${ }^{1, *}$ Monireh Asadi Abchouyeh ${ }^{2}$ \\ ${ }^{1}$ Department of Mathematics Khajeh Nasir ad-din Toosi University of Tecnology, Tehran, Iran \\ azakeri@kntu.ac.ir \\ ${ }^{2}$ Payam-e-Noor University, Esfahan, Iran \\ m.asadi8689@gmail.com
}

Received: February 2012, Revised: May 2012

Online Publication: July 2012

\begin{abstract}
In this paper, a constrained optimal control problem is considered where constraint is elliptic partial differential equations of second order together with the boundary condition of Dirichlet type. The main purpose is detecting an appropriate solution for control and state function by using boundary element method in order to discretized PDEs. In this way, first a quadratic objective, linear constraints optimization problem rewritten respected to main problem, next it can be solved numerically with the help of appropriate solution algorithms, which should exploit the structure of the problem, we solved it by generalized Newton's method. Some numerical experiments obtained by using boundary element method (BEM) and finite element method (FEM) are given in the final section of this paper.
\end{abstract}

Keywords: Boundary element method, optimal control problem, Poisson equation, Elliptic equation, Finite element method.

\section{Introduction}

Solving optimization problems subject to constraints given in terms of PDE with additional constraints on the control and/or states in one of the most challenging problems in the context of industrials and economical applications, where the transition from model-based numerical simulations to model-based design and optimal control is crucial. For the treatment of such optimization problems the interaction of optimization techniques and numerical simulations plays a central role [1]. 
This project is concerned with the application of an innovative mathematical technique for solution of constrained optimization problems where a PDE or a system of PDE is constraints. The problem that we investigate is an optimal control problem.

In fact generalization and development of appropriate methods of the calculus of variations has rised optimization argument. In 1950, it is largely due to the work of Richard Bellman in the United States. Early 1960, Lev Pontryagin and his collaborators in the Soviet Union have registered the Pontryagin's maximum principle [2]. Optimal control problem are generally nonlinear and therefore, generally do not have analytic solutions. As a result, it is necessary to employ numerical methods to solve optimal control problems. In the early years of optimal control (circa 1950s to 1980s) the favored approach for solving optimal control problems was that of indirect methods. In an indirect method, the calculus of variations is employed to obtain the firstorder optimality conditions. These conditions result in a two-point (or, in the case of a complex problem, a multi-point) boundary-value problem. This boundary-value problem actually has a special structure because it arises from taking the derivative of a Hamiltonian. The approach that has risen to prominence in numerical optimal control over the past two decades (from the 1980s to the present) is that of so called direct methods. In a direct method, the state and/or control is approximated using an approximated using an appropriate function approximation. Simultaneously, the cost functional is approximated as a cost function. Then, the coefficients of the function approximation are treated as optimization variables and the problem is transcribed to a nonlinear optimization problem. Depending upon the type of direct method employed, nonlinear optimal control problem can be quite small or may be large. Optimal control problems with PDE constraints are very important in practical applications but are very costly numerical solving.

In 2004, M. Weizer, A. Schiela and T. Ganzler offered a primal interior point method for optimization with PDE constraints. Pointwise elimination of the control leads to a homotopy in the remaining state and dual variables. The a priori elimination of the least regular control permits to obtained accuracy with comparatively coarse meshes which requires fine meshes and thus leads to large finite dimensional subproblems [4]. As a different approach to alleviate the need for mesh refinement Hinze suggested to eliminate the control $u$ analytically from the optimality system by computing it from the dual variable $\lambda$. For certain class of optimal control problems this is a simple pointwise calculation [6]. In 2010, D. Clever and J. Yang applied a projected gradiant method with Armijo step size to determine an optimal boundary control considering the first wolf condition [5].Optimal control problem with linear state equation and quadratic objective function are called linear-quadratic [1].

In this article, an optimal control problem with PDE constraints proposed by Hinze is concentrated and obtained an approximate solution. With respect constraints of problem it is worthy to estimate the approximated solution by applying numerical methods.

\begin{tabular}{|ll|}
\hline \multicolumn{1}{|l|}{ Nomenclatures } \\
$\Omega$ & Computation domain \\
$Y=H_{0}^{1}(\Omega)$ & Sobolev space \\
$H, U$ & Hilbert space \\
$R, Z$ & Banach space \\
$Y_{a d}, U_{a d}$ & Convex and closed subspaces $Y, U$ \\
\hline
\end{tabular}




$\begin{array}{ll}L(U, Y) & \text { Space of all linear operators } A: U \rightarrow Y \\ \Delta & \text { Laplace operator }\end{array}$

\section{Constrained optimal control problem}

We consider $y_{0}$ denotes the known function of $u \in U_{a d}, \quad \alpha>0$ denotes a known constant and $y, u$ are respectively unknown state and control functions that by finding them the functional $J(y, u)$ of the form

with the following constraints

$$
\operatorname{Min}_{(y, u) \in Y \times U} J(y, u):=\frac{1}{2}\left\|y-y_{0}\right\|_{L^{2}(\Omega)}^{2}+\frac{\alpha}{2}\|u\|_{U}^{2} \quad(a-2-1)
$$

$$
\begin{cases}-\Delta y=u, & \text { in } \Omega, \\ y=0 & \text { on } \partial \Omega, \\ \text { and } & \\ u \in U_{a d} \subset U & (b-2-1)\end{cases}
$$

Minimize in computational domain $\Omega$.

This problem can be converted to a linear-quadratic optimization problem of the form

$$
\begin{aligned}
& \min _{(y, u) \in Y \times U} J(y, u):=\frac{1}{2}\left\|Q y-q_{d}\right\|_{H}^{2}+\frac{\alpha}{2}\|u\|_{U}^{2} \\
& \text { subject to } \quad A y+u=g, \quad u \in U_{a d}, y \in Y_{a d} .
\end{aligned}
$$

Where $q_{d} \in H, g \in Z, A \in L(Y, Z), Q \in(Y, H)$ and the following assumption holds.

\section{2-1 Existence Result for a General Linear-Quadratic Problem Assumption}

1. $\alpha \geq 0, U_{a d} \subset U$ is convex, closed and in the case $\alpha=0$ bounded.

2. $Y_{a d} \subset Y$ is a convex and closed, such that (3-1) has a feasible point.

3. $A \subset L(Y, Z)$ has a bounded inverse.

Definition A state-control pair $(\bar{y}, \bar{u}) \in Y_{a d} \times U_{a d}$ is called optimal for (3-1), if $A \bar{y}+\bar{u}=g$ and

$$
J(\bar{y}, \bar{u}) \leq J(y, u) \quad \forall(y, u) \in Y_{a d} \times U_{a d}, A y+B u=g .
$$

Theorem Let assumption hold. Then problem (3-1) has an optimal solution $(\bar{y}, \bar{u})$. If $\alpha>0$ then the solution is unique.

Proof [1].

\section{A Numerical Method for Finding a Optimal Solution}


In order to tackle $(2-1)$ numerically has discussed two different approaches. The first is called First discretiez, then optimize, the second First optimize, then discretize.

In this paper we will use first approach for solving problem. For this purpose, first we approximate domain $\Omega$ with an arbitrary polygon, not necessarily regularly, using boundary element method. Then let us display coordinate of $j$ th vertex of polygon by $\left(x_{1 j}, x_{2 j}\right) j=1,2, \ldots, N$. In this case, $y$ and $q$ are respectively function and passing flux through the border of region $\Omega$. With choosing boundary element method with linear, continuous elements for $y$, we convert Poisson equation into the form

$$
y^{(i)}+\int_{\Gamma} y q^{*} d \Gamma+\int_{\Omega} u y^{*} d \Omega=\int_{\Gamma} y^{*} q d \Gamma, \quad i=1,2, \ldots, M
$$

Such that

$$
y^{*}=\frac{1}{2 \pi} \ln \frac{1}{r}
$$

and

$$
r=\sqrt{\left(x_{1}-x_{1 i}\right)^{2}+\left(x_{2}-x_{2 i}\right)^{2}}
$$

$\left(x_{1 i}, x_{2 i}\right)$ denotes interior or border point and $y^{(i)}$ represent amount of function $y$. Hence, we get the linear system $A y=E s$ by using the linear continuous elements and approximation of integrals.

Then function $y_{h}$ denotes approximated $y$ and is represented of the form $y_{h}\left(x_{1 i}, x_{2 i}\right) i=1,2, \ldots, M$. Let $A=\left\{u_{1}, u_{2}, \ldots, u_{m}\right\}$ denotes a linearly independent set so that $u_{i}$ is represented of the form $x_{1}^{k_{1}} x_{2}^{k_{2}}$ for non-negative and integer $k_{1}, k_{2}$ and $U_{d}=S p a n A$.

Finally let $Z_{h}:=I_{h} Z=\sum_{i=1}^{M} z_{i} \Phi_{i}$ where $I_{h}: L^{2}(\partial \Omega) \rightarrow W_{h}$ denotes a continuous interpolation operator, $W_{h}$ denotes space of piecewise linear functions with step size $h$ on $\partial \Omega, \Phi_{i}$ denote piecewise linear functions. We set $U_{a d}^{d}=U_{a d} \cap U_{d}$. It is convenient to assume that $U_{a d}^{d}$ may be represented in the form

$$
U_{a d}^{d}=\left\{u \in U, u=\sum_{j=1}^{m} s_{j} u_{j}, s_{j} \in \mathbb{R}\right\} .
$$

Now we replace problem (3-1) by

$$
\left\{\begin{array}{l}
\min _{\left(y_{h}, u_{d}\right) \in Y_{h} \times U_{d}} J(y, u):=\frac{1}{2}\left\|y-z_{h}\right\|_{L^{2}(\Omega)}^{2}+\frac{\alpha}{2}\left\|u_{d}\right\|_{U}^{2} \\
\text { s.t.A } y=E s \quad s \in \mathbb{R} . \\
u_{d} \in U_{a d}^{d} .
\end{array}\right.
$$

This is now a finite dimensional optimization problem with quadratic objective, linear equality constraints. Since $A$ is an invertible matrix and thus regular problem $(3-1)$ is equivalent to minimizing the reduced functional $\hat{J}(s)=J\left(A^{-1} E s, s\right)$ over the set $\mathbb{R}$.

Due to theorem problem (1-2) admits a unique solution $(\bar{y}, \bar{u}) \in Y_{a d} \times U_{a d}$.

In next section we investigate a numerical method based on discretization methods afforded by Hinze in [1].

\section{A Numerical Example}

In this section, we discuss a numerical example illustrating the behavior of our method and in particular compar the results obtained with the finite element method obtained by Hinze in [1]. Consider the following optimization problem: 


$$
\left\{\begin{array}{l}
\underset{(y, u) \in Y \times U}{\operatorname{Min}} J(y, u):=\frac{1}{2}\left\|y-y_{0}\right\|_{L^{2}(\Omega)}^{2}+\frac{\alpha}{2}\|u\|_{U}^{2}, \\
\text { s.t. }\left\{\begin{array}{l}
-\Delta y=0 \quad \text { in } \Omega, \\
y=B u \quad \text { on } \partial \Omega \\
\text { and } \\
u \in U_{a d} \subseteq U
\end{array}\right.
\end{array}\right.
$$

Here consider $\alpha=1, y_{0}=-\operatorname{sign}\left(x-0 / 5-0 / 1 \pi^{-1}\right), \Omega=[0,1] \times[0,1], U=\mathbb{R}^{2}$ and $y, u$ are respectively state and control function. Consider $u\left(x_{1}, x_{2}\right)=s_{1}+s_{2} x_{1}+s_{3} x_{2}+s_{4} x_{1} x_{2}$ where $s_{1}, s_{2}, s_{3}, s_{4}$ are unknown constant coefficients. Then solve Poisson equation $-\Delta y=u$ with mixed boundary condition on border $\partial \Omega$ by using boundary element method with step size $h=0.2$ and determine different values $y$ depending on unknown quantities $s_{1}, s_{2}, s_{3}, s_{4}$ (Table 1). By replacing obtained $y$ and $u\left(x_{1}, x_{2}\right)=s_{1}+s_{2} x_{1}+s_{3} x_{2}+s_{4} x_{1} x_{2}$ in functional $J(y, u)$ we get a nonlinear function with $s_{1}, s_{2}, s_{3}, s_{4}$ variables. In order to minimizing functional $J(y, u)$ we use iterative Newton method and obtain unknown values $s_{1}, s_{2}, s_{3}, s_{4}$. Also we obtain values of state $y$ in inner grids.

Table 2 shows the numerical results for $s_{1}, s_{2}, s_{3}, s_{4}$, control function $u$ and optimal value of $J(y, u)$.

Table 3 shows values of state $y$ in inner grids.

Table 1. Different values of state $y$ in grids $(0.8,0.2 i)(i=1,2,3,4)$ for mesh size $h=0.2$ by BEM and FEM.

\begin{tabular}{|c|c|c|}
\hline$x_{2}$ & $y(\mathrm{BEM})$ & $y$ (FEM) \\
\hline 0.2 & $0.09 s_{1}+0.057 s_{2}-0.033 s_{3}-0.021 s_{4}$ & $0.04 s_{1}+0.024 s_{2}+0.008 s_{3}+0.004 s_{4}$ \\
\hline 0.4 & $0.09 s_{1}+0.06 s_{2}-0.043 s_{3}-0.027 s_{4}$ & $0.04 s_{1}+0.024 s_{2}+0.016 s_{3}+0.009 s_{4}$ \\
\hline & & $0.04 s_{1}+0.024 s_{2}+0.024 s_{3}+0.014 s_{4}$ \\
\hline 0.6 & $0.09 s_{1}+0.06 s_{2}-0.051 s_{3}-0.032 s_{4}$ & \\
\hline 0.8 & $0.09 s_{1}+0.057 s_{2}-0.057 s_{3}-0.036 s_{4}$ & $0.04 s_{1}+0.024 s_{2}+0.032 s_{3}-0.019 s_{4}$ \\
\hline
\end{tabular}


Ali Zakeri, Monireh Asadi Abchouyeh/ TJMCS Vol. 4 No. 3 (2012) 371 - 378

Table 2. Control function $u$ for different iteration by iterative Newton method with mesh size $h=0.2$ by BEM.

\begin{tabular}{|c|c|c|c|c|c|c|}
\hline \multirow{2}{*}{$x_{2}$} & \multicolumn{3}{|c|}{ Different values of $s_{1}, s_{2}, s_{3}, s_{4}$} & \multirow{2}{*}{$u\left(x_{1}, x_{2}\right)$} & \begin{tabular}{c} 
optimal values \\
of \\
\cline { 2 - 6 }
\end{tabular} \\
\cline { 2 - 6 } & $s_{1}$ & $s_{2}$ & $s_{3}$ & $s_{4}$ & $J(y, u)$ \\
\hline 0.2 & 0.3743 & 0.2482 & -0.0399 & -0.2083 & $0.03743+0.2482 x_{1}-0.0399 x_{2}-0.2083 x_{1} x_{2}$ & 0.49403 \\
\hline 0.4 & 0.275 & 0.1866 & -0.0131 & -0.0734 & $0.0257+0.1866 x_{1}-0.01317 x_{2}-0.07345 x_{1} x_{2}$ & 0.49445 \\
\hline 0.6 & 0.0125 & 0.113 & 0.0131 & 0.0735 & $0.0125+0.1131 x_{1}+0.01314 x_{2}+0.07350 x_{1} x_{2}$ & 0.49445 \\
\hline 0.8 & -0.0024 & 0.0399 & 0.0399 & 0.2083 & $-0.0024+0.0399 x_{1}+0.0398 x_{2}+0.2083 x_{1} x_{2}$ & 0.49403 \\
\hline
\end{tabular}

Table 3. Different values of state $y$ in grids $(0.8,0.2 i)(i=1,2,3,4)$ for mesh size $h=0.2$ by FEM and BEM.

\begin{tabular}{|c|c|c|}
\hline$x_{2}$ & $y$ (BEM ) & $y$ (FEM) \\
\hline 0.2 & 0.0119237 & -0.0022 \\
\hline 0.4 & 0.0110898 & -0.0029 \\
\hline 0.6 & 0.0110898 & -0.0029 \\
\hline 0.8 & 0.0119236 & -0.0022 \\
\hline
\end{tabular}




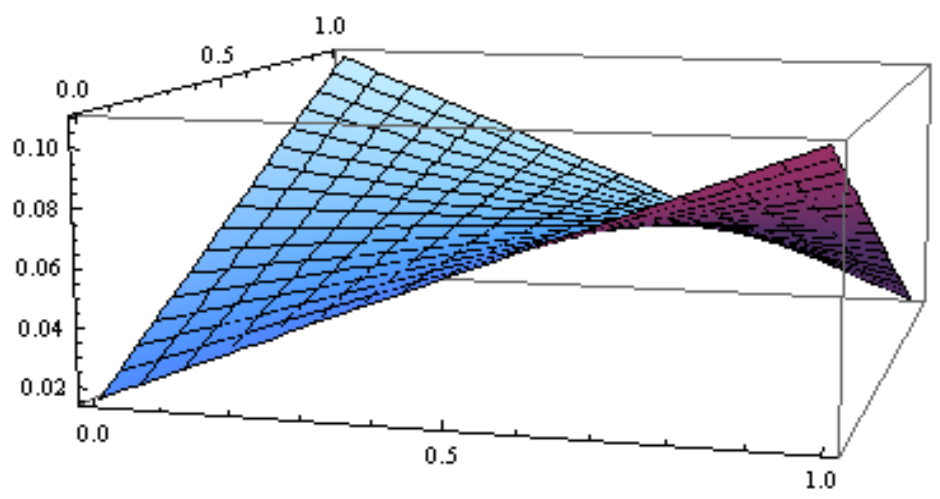

Figure 1. Function $u\left(x_{1}, x_{2}\right)$ in interval $[0,1]$ for tenth iterative by Newton method with mesh size $h=0.5$.

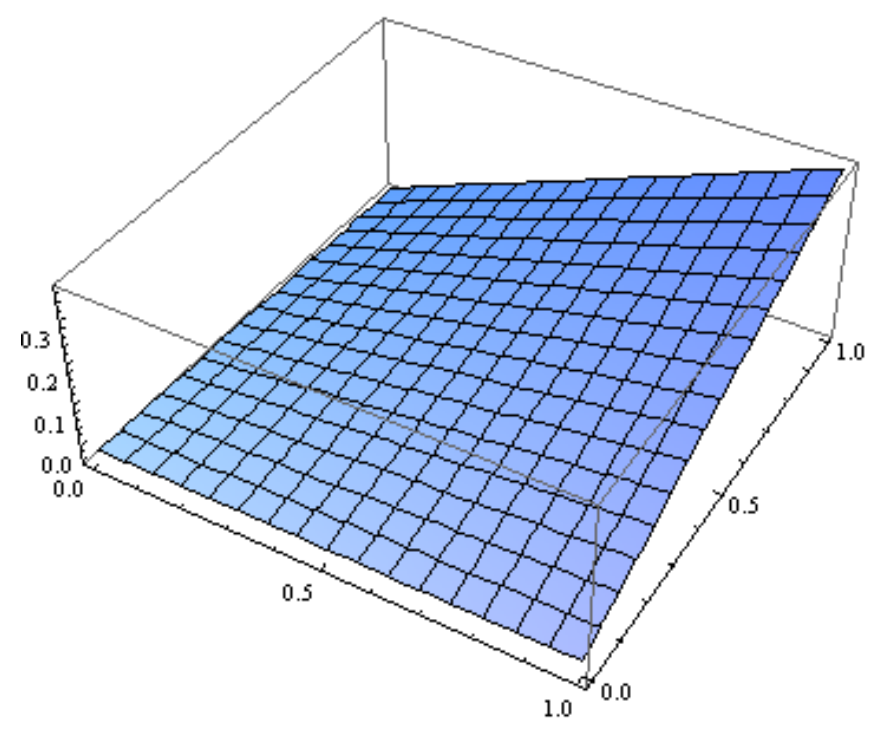

Figure 2. Function $u\left(x_{1}, x_{2}\right)$ in interval $[0,1]$ for tenth iteration by Newton method with mesh size $h=0.2$

\section{Conclusion}

PDE constrained optimization problems arise in many applications. Evaluation of objective function and constraint function involves expensive simulations. The robust and efficient solution of such optimization problems requests the integration application specific structure numerical simulation and optimization algorithm. In this paper, we have presented an elliptic optimal control proplem and computed approximation solution with two numerical computational method of solving linear partial differential equations FEM and BEM. The advantages in the boundary element method arise from the fact that only the boundary (or boundaries) of the domain of the PDE requires sub-division to produce a surface or boundary mesh [8]. The use of the Green-Guass theorem and a fundamental solution formulation means that the BEM involves no approximation of the differential equation in the domain-only in its approximations of the boundary conditions but in FEM differential equation is being approximated [3]. With mesh size $h=2^{-6}$ and using the finite element method we got the objective function value $J(y, u)=0.474737$ and with mesh size $h=0.2$ and 
using the boundary element method we got the objective function value $J(y, u)=0.494455$. Then the boundary element method is more efficient than other methods, including finite element, in terms of computational resources for problem where there is a small surface/volume ratio.

\section{Reference}

[1] Hinze, M., Pinnau R., Ulbrich M., Ulbrich S., "Optimization with PDE constraints", -ISBN 978-14020-8839-1. 2009.

[2] Pontryagin L. S., "The Mathematical Theory of Optimal Processes". 1962

[3] Hunter, P,. Pullan, A,. "HFEM/BEM NOTES",. February 21,2001.

[4] Weiser M., Ganzler T.,Schtela A. " A control reduced primal interior point method for PDE constrained optimization". ZIB Report 04-38, Zuse Institute Berlin, 2004.

[5] Clever D, Lang J. "Optimal control of radiative heat transfer in glass cooling with restrictions on the temperature gradient". Optim. Control Appl. Math, 2011.

[6] Hinze, M., "A generalized discretization concept for optimal control problem with control constraints", Comput, Optim, Apple, 2009. 